\title{
Gradhiva
}

GRADHIV

Revue d'anthropologie et d'histoire des arts

$1 \mid 2005$

Haîti et l'anthropologie

\section{Entretien avec René Depestre}

Jean-Luc Bonniol

\section{OpenEdition \\ Journals}

Édition électronique

URL : http://journals.openedition.org/gradhiva/261

DOI : 10.4000/gradhiva.261

ISSN : 1760-849X

\section{Éditeur}

Musée du quai Branly Jacques Chirac

\section{Édition imprimée}

Date de publication : 1 mai 2005

Pagination : $31-45$

ISBN : 2-915133-08-5

ISSN : 0764-8928

Référence électronique

Jean-Luc Bonniol, « Entretien avec René Depestre », Gradhiva [En ligne], 1 | 2005, mis en ligne le 10 décembre 2008, consulté le 01 mai 2019. URL : http://journals.openedition.org/gradhiva/261 ; DOI 10.4000/gradhiva.261

Ce document a été généré automatiquement le 1 mai 2019.

(c) musée du quai Branly 


\title{
Entretien avec René Depestre
}

\author{
Jean-Luc Bonniol
}

\section{NOTE DE L'ÉDITEUR}

Il nous a semblé intéressant de faire suivre cette conférence d'un certain nombre d'extraits de l'entretien que René Depestre a accordé à Jean-Luc Bonniol, en avril 2004. Les extraits ont trait aux rôles joués par quelques grandes figures d'intellectuels d'Haïti ou d'ailleurs et de la place de l'anthropologie dans la construction de la Nation haïtienne. Toutes réflexions que l'on retrouve réverbérées dans le dossier proposé ci-après. L'entretien dans son ensemble sera prochainement publié sous la forme d'un ouvrage qui retracera l'itinéraire littéraire et les engagements politiques de René Depestre.

Pour garder sa fluidité et son oralité au texte, nous avons, dans la mesure du possible, laissé de côté les questions qui le ponctuent. 
...J'ai conscience qu'Haïti a pris un mauvais chemin dans l'histoire. C'est là une conscience qui a été très précoce. Longtemps avant moi, dès la fin du XIX ${ }^{\mathrm{e}}$ siècle, les premiers intellectuels haïtiens ont eu à exprimer leur inquiétude à cet égard. On pouvait déjà tenir le terrorisme d'État contre le corps civique haïtien pour un phénomène récurrent de la vie publique. Louis-Joseph Janvier, diplômé de la Faculté de médecine de Paris mais qui ne s'intéressait pas seulement à la médecine, devait porter des jugements très sévères sur l'état de déliquescence des mœurs politiques du pays. Il attira l'attention sur les dangers qu'il y aurait à persévérer dans

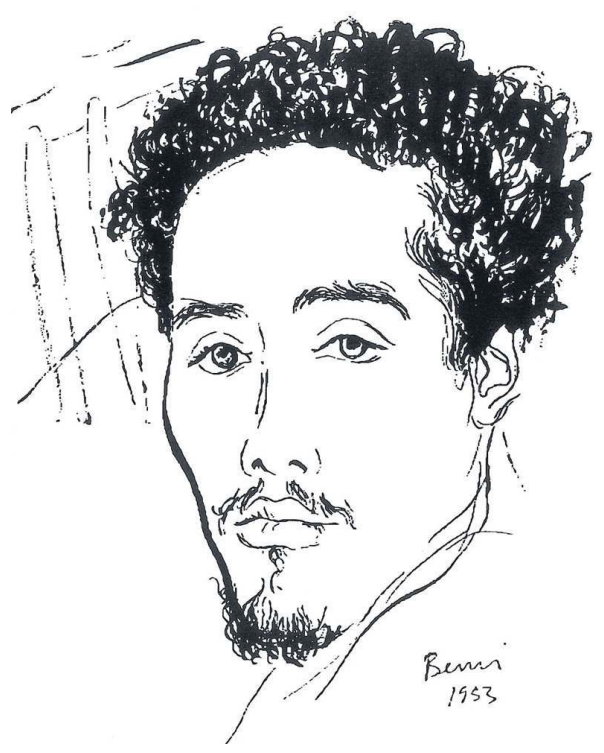
la voie de tyrannie ou de satrapie caractéristique de la scène "nationale » d'Haïti. Son contemporain et ami, l'éminent publiciste Anténor Firmin, était intervenu dans le même sens dans ses écrits, notamment dans un livre intitulé De l'égalité des races humaines, publié à Paris, en réponse aux thèses racistes du comte de Gobineau. Un autre homme de culture, Edmond Paul, économiste renommé, avait également alerté l'opinion, avec autant de vigueur que Frédéric Marcelin, le fondateur de l'art narratif d'expression française en Haïti. Toutes ces voix averties - à la veille du centenaire de l'indépendance d'Haïti (1904) - avaient signalé que le pays allait tout droit à la catastrophe qu'il subit aujourd'hui. 


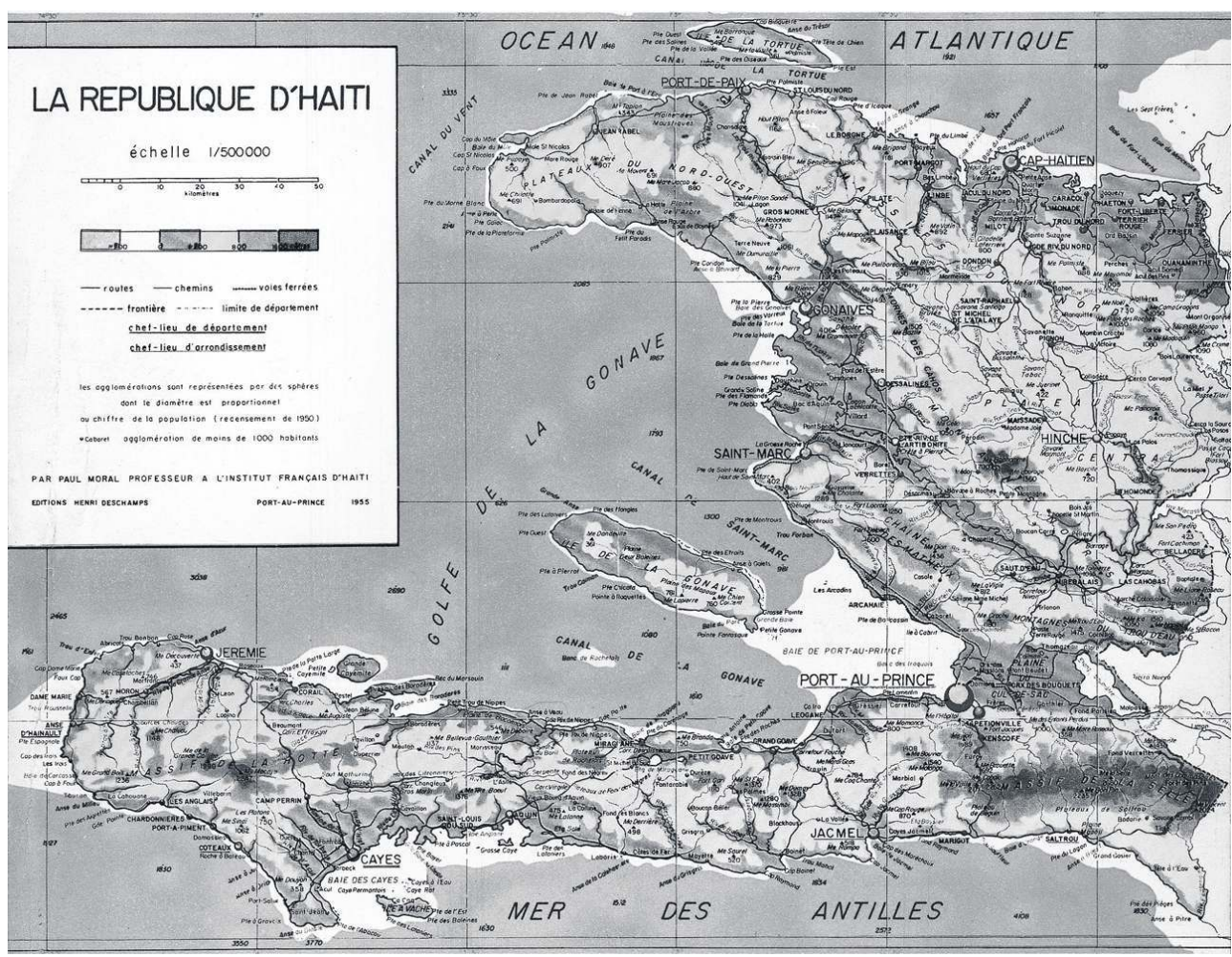

droits réservés

2 Lors du centenaire de l'indépendance d'Haïti, lors de la première célébration de cette indépendance, en 1904, il y a eu beaucoup de gens, notamment Rosalvo Bobo, qui ont tiré la sonnette d'alarme et qu'on n'a pas écoutés. Et cela a continué jusqu'à l'occupation américaine qui aura été un avertissement, cette fois-ci n'émanant pas seulement d'intellectuels, mais des faits eux-mêmes, puisque le pays qui, le premier, s'était débarrassé des chaînes de l'esclavage, se trouvait occupé par les forces de Marines des États-Unis. Cela a été un coup très rude pour le patriotisme haïtien parce que, au milieu de toute cette satrapie qui a prédominé dans les mœurs politiques, il y a toujours eu parallèlement un sentiment patriotique, très charnel, lié à la terre d'Haïti, à l'amour d'Haïti, une sorte de dévotion presque religieuse aux luttes que nos ancêtres avaient menées de 1791 jusqu'à 1804. Mais finalement, avec le recul, on s'aperçoit que, au fond, tout cela était une rhétorique patriotarde qui n'a jamais eu de prise sur le peuple haïtien lui-même, sur la paysannerie particulièrement, et que le fossé s'est creusé de plus en plus entre la paysannerie qui s'est constituée au $\mathrm{XIX}^{\mathrm{e}}$ siècle et «l'élite », la prétendue élite dirigeante du pays.

3 En réaction à l'occupation américaine, il y a eu en Haïti un réveil à partir de 1920 incarné d'abord par Jean Price-Mars, qui a condamné cette tendance, qui prévalait dans la vie publique haïtienne, à mépriser l'Afrique, à l'oublier. En même temps que des hommes comme Anténor Firmin et Joseph Janvier inculquaient aux Haïtiens la fierté de leur « race » - on reviendra sur cette affaire de « race » plus longuement -, il y avait des gens, notamment parmi l'élite dirigeante, qui faisaient tout pour qu'on oublie l'Afrique, qu'ils tenaient pour une terre de barbarie, tandis qu'Haïti, grâce à son élite latine, était le phare de la latinité aux Caraïbes, ce qui était une imposture pure et simple. Price-Mars a écrit 
un livre qui a secoué la conscience haïtienne, Ainsi parla l'oncle, qui était une tentative de réhabilitation de la véritable Afrique, de l'Afrique colonisée. Il a eu l'appui d'une nouvelle génération qui a fait face à l'occupation américaine, qu'on appelle «la génération de l'occupation", constituée, outre de Jean Price-Mars, de Jacques Roumain, de Carl Brouard, de Philippe Thoby-Marcelin, d'Émile Roumer et, peu de temps après, d'Anthony Lespès, de Jean Brière, c'est-à-dire toute une pléiade de jeunes gens qui ont constitué ce qu'on appelle «le mouvement indigéniste ».

4 Le mouvement indigéniste était un mouvement politique parce qu'il avait un côté nationaliste, il luttait contre l'occupation américaine et en même temps ses membres essayaient de rénover les lettres haïtiennes en s'adossant directement aux prédécesseurs, c'est-à-dire à des hommes comme Frédéric Marcelin, Fernand Hibbert, Antoine Innocent... Si on veut faire un bilan, je pense qu'on peut considérer le mouvement indigéniste comme un mouvement équivalent de la négritude et de l'indigénisme sudaméricain (pro-indien). C'est à peu près le même état d'esprit: accompagner la célébration des lettres et des arts dans le pays d'un retour à l'Afrique, voir dans le continent africain une source identitaire qui pouvait enrichir les conditions locales haïtiennes de la création artistique et de la création littéraire. Cela représentait une prise de conscience importante, avec une certaine connotation raciale, dans la mesure où on réhabilitait la "race noire ", mais dans le même temps on sortait la littérature haïtienne de l'imitation pure et simple des écoles littéraires de Paris, on inculquait à la jeunesse le sentiment qu'Haïti valait la peine d'être célébrée pour l'originalité de son histoire, pour la beauté de ses paysages, pour ses spécificités tant physiques que poétiques et morales. À ce titre, le mouvement indigéniste se trouve au fondement de la littérature haïtienne moderne. Ma génération a ensuite pris le relais, avec Jacques Stephen Alexis, Roger Gaillard, et d'autres, Lucien Lemoine, Felix Morisseau-Leroy.

5 Il y a donc toujours eu, face à la satrapie haïtienne, une intelligentsia. D'abord une intelligentsia d'historiens, des gens comme Beaubrun Ardouin, qui a été un historien important, Saint-Rémy des Cayes, Thomas Madiou, qui a été le principal historien du XIX siècle. Ces gens-là, déjà, ont eu une vision de ce que pouvait être Haïti. Ils réfléchissaient sur la nation haïtienne. Mais il y avait un malentendu grave parce que, au fond, et aujourd'hui on peut le voir avec clarté, il n'y a jamais eu véritablement de nation haïtienne. Cela montre que l'histoire n'est pas univoque, que le processus qui a conduit, en Europe, à la constitution de la Nation, à travers la Renaissance, l'Encyclopédie, les Lumières et qui, grâce à la Révolution française, a débouché sur la République et a créé des vertus républicaines, n'est peut-être pas la seule voie de l'histoire. Mais, du fait de sa naissance dans l'histoire de la France, on pouvait penser qu'Haïti était capable de faire le saut de la plantation esclavagiste à la République. Et c'est cela qui ne s'est pas passé. Il faudrait chercher pourquoi Haïti n'a pas pu devenir une république, parce qu'une république suppose la démocratie, suppose des institutions démocratiques, une société civile, une civilité démocratique. Cela n'a jamais pris en Haïti.

6 L'intelligentsia en a eu conscience, mais comme c'est un pays qui n'a pas été alphabétisé, très peu de gens finalement lisaient les auteurs haïtiens importants comme Janvier, Anténor Firmin, Price-Mars, Jacques Roumain. Cette littérature plutôt contestataire circulait dans l'élite qui en faisait rapidement une sorte de décoration, mais jamais un aliment pour la constitution d'une nation. On n'a pas pu constituer un État-nation. C'est là le fond du problème : aujourd'hui, au moment où Aristide vient de partir, il n'y a toujours pas de nation haïtienne, ni d'État-nation digne de ce nom. 
On peut dire que Price-Mars est le disciple spirituel d'Anténor Firmin et de Louis-Joseph Janvier. C'est de là qu'il vient. Très jeune, il a fait sa médecine à Paris ; quand il est rentré en Haïti, il a été très choqué par le dédain dans lequel les représentants de l'élite haïtienne tenaient tout ce qui était africain. Or, on ne peut pas comprendre le vodou si on ne se réfère pas à l'Afrique, on ne peut pas comprendre la langue créole si on ne se réfère pas à l'Afrique. On ne peut pas comprendre la mentalité haïtienne, les danses haïtiennes, les pratiques haïtiennes de la vie si on n'a pas en tête une connaissance de l'héritage africain. Or l'élite haïtienne faisait tout, et ce d'autant plus après l'arrivée des Américains qui apportaient de l'eau à son moulin, pour qu'on ignore l'Afrique. Price-Mars a étudié d'abord le folklore haïtien; il y a trouvé des similitudes entre la danse, le carnaval, les contes populaires, qu'on ne pouvait pas comprendre si on ne se référait pas à l'expérience africaine de la vie. Mais il a compris aussi que, après plus d'un siècle de développement en Haïti, il y avait une " haïtianité », qu'il y avait ce qu'on appelle aujourd'hui une identité haïtienne, qu'il y avait une " créolité ", si on peut dire, qui ne recouvrait pas seulement la langue, mais aussi les mœurs, la vie sociale, les familles, la danse, la musique et la religion.

8 À partir de là, il a fait, sous une forme très agréable, des conférences qu'il a appelées Ainsi parla l'oncle, comme si c'était un conte qu'un vieil oncle racontait aux Haïtiens. Cela a eu un retentissement énorme, le livre a aussi été bien lu à l'étranger, cela a contribué au renouveau du mouvement indigéniste et a coïncidé avec le retour de Jacques Roumain qui venait de faire ses études en Europe.

Price-Mars a par la suite atteint un statut considérable dans la vie intellectuelle haïtienne. Pour ma génération, c'était l'intellectuel le plus important. J'ai voulu suivre ses cours, ce que j'ai fait pendant quelque temps parce qu'il venait de créer le Bureau d'ethnologie, avec Jacques Roumain, qui était de retour, après avoir été expulsé d'Haïti en 1935. À l'époque, j'étais au lycée mais cela m'intéressait déjà. Je suivais les manifestations du Bureau d'ethnologie.

10 [...] Le Bureau d'ethnologie, c'était une entreprise modeste dotée d'un département archéologique grâce à Métraux qui se trouvait en Haïti. Mabille est arrivé, il a renforcé l'équipe. On a fait venir Césaire. Il s'est constitué un tissu intellectuel extrêmement intéressant autour de Roumain et de Price-Mars. Roumain est mort en 1944. Price-Mars a vécu beaucoup plus longtemps...

[...]

Dans mes premiers textes sur Price-Mars, j'ai voulu rendre un hommage à l'homme. J'ai été très sévère à son égard tout en ayant beaucoup d'admiration pour lui parce qu'il m'avait tendu la main quand j'ai publié mes premiers poèmes. Il m'a accueilli avec une grande affection après la mort de Jacques Roumain. J'étais très fier, si jeune, d'être proche de lui, mais quand j'ai vu l'usage que ses amis du Bureau d'ethnologie faisaient de sa pensée... Price-Mars ne savait sur quel pied danser avec eux, parce qu'ils le flattaient beaucoup et qu'ils se disaient très proches de lui. Price-Mars était plutôt un homme reconnu par toute la nation haïtienne, y compris par l'élite; il avait épousé une mulâtresse, il habitait à Pétionville, il était reçu dans tous les salons... Ce n'était pas son problème. Il ne pensait pas comme ces gens. Il transcendait la question «raciale ». Il a certes manqué de courage en 1930 pour affronter vraiment Vincent dans l'arène. Il a été très prudent, il s'est retiré. Car ce n'était pas un révolutionnaire, c'était un homme de 
cabinet, très doux, très gentil, très instruit... Un homme admirable. Mais les duvaliéristes l'ont utilisé et il a fait preuve de complaisance, il ne les a pas attaqués.

Marché de Port-au-Prince

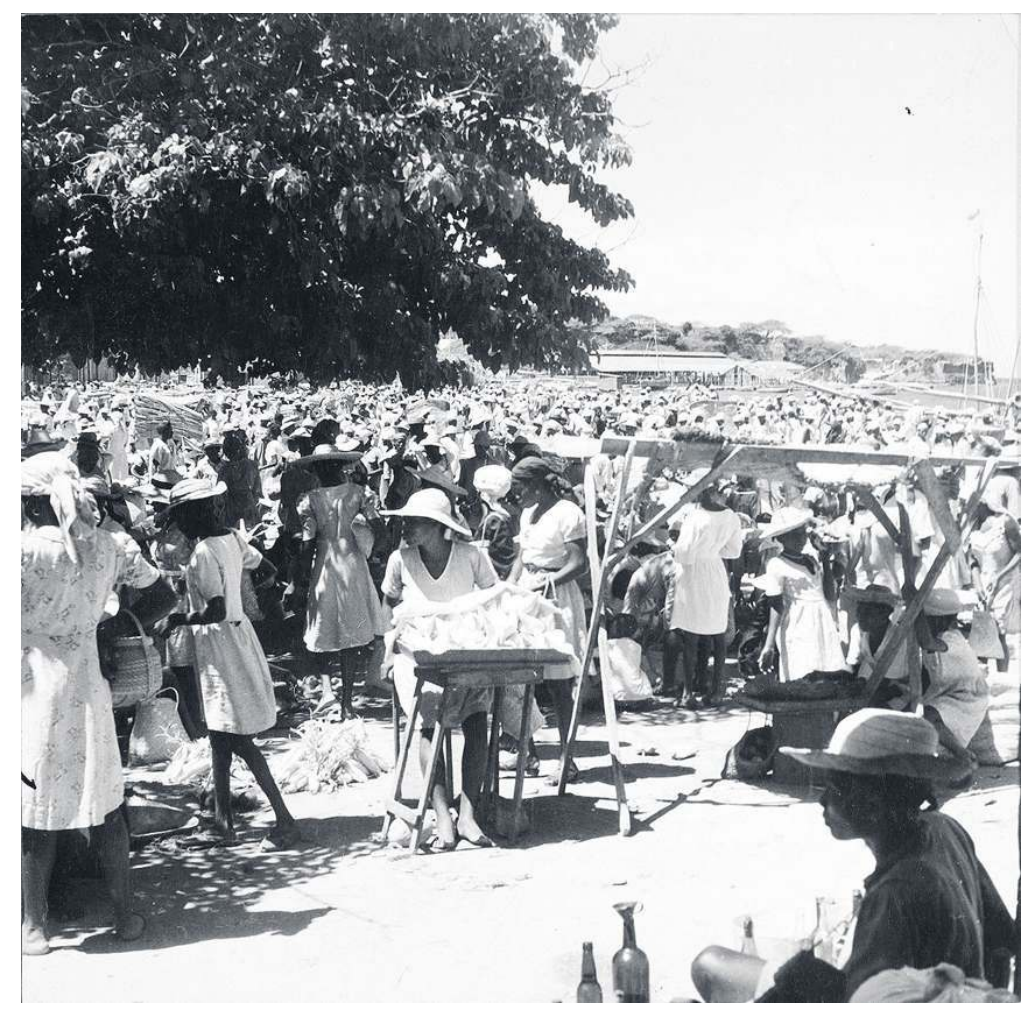

(c) musée du quai Branly

12 Jusqu'au moment où Piquion a écrit un petit livre. Là, il a tenu à faire une déclaration pour dire qu'il se démarquait des thèses de Piquion, que ce n'était pas exactement sa pensée. Il faut lui rendre cette justice : avant de mourir, il a eu le temps de réagir. Et les duvaliéristes, sur ses vieux jours, l'ont isolé tout en lui rendant beaucoup d'hommages, en lui faisant des funérailles nationales... Mais finalement, ils ont senti que Price-Mars n'appartenait pas à la même école qu'eux. Price-Mars était beaucoup plus proche, sans être communiste, de Jacques Roumain. C'était un homme de gauche. Quand j'ai publié mes premiers poèmes, qui étaient des poèmes plutôt libertaires, contestataires, qui voulaient en découdre avec toute la société dominante haïtienne, Price-Mars m'a protégé, reçu, aidé. Il m'a défendu.

[...]

C'est lui qui a inventé la notion de bovarysme collectif. Il a toujours été très sévère pour l'élite haïtienne, mais il pensait qu'il y avait dans cette élite des gens remarquables. Il y avait un homme comme Léon Laleau, comme le père de Jacques Alexis, Stephen Alexis, qui était un intellectuel éminent. Il y avait des hommes de valeur. Dantès Bellegarde n'était pas un imbécile, c'était même un homme remarquable. Il y avait des mulâtres intelligents comme Seymour Pradel, par exemple, qui n'ont pas partagé les préjugés de couleur de l'élite dominante.

[...]

Même l'apparition d'un homme [comme Jacques Roumain], dans la vie d'un pays, dans le peu de temps qu'il a vécu, montre que ce pays a plus à offrir que la sorte de surplace 
existentiel qui a gelé son développement. Comme le disait Jacques Alexis, Jacques Roumain est l'exemple vivant qu'un pays qui produit un tel homme, ne peut pas mourir. C'est le sentiment que, très jeunes, Alexis et moi, nous avons eu en fréquentant très brièvement Roumain.

Jacques Roumain avait lu Marx et Engels dans le texte à Zurich. Il était très curieux, c'était un garçon originaire de la bourgeoisie haïtienne, de la bourgeoisie sucrière, mais qui avait une très grande sensibilité et qui, parce qu'il passait ses vacances d'enfant riche à la campagne, avait connu la vie paysanne. Jacques Roumain a tout de suite compris la parole de Price-Mars et il a fondé la Revue Indigène dans les mêmes années, en 1927-1928. Il n'avait pas 25 ans, il était déjà célèbre, il a écrit des nouvelles, des contes, et il a radicalisé sa pensée à mesure que le régime de Stenio Vincent le décevait, que son nationalisme était trahi.

Roumain a voulu étudier l'ethnologie à son tour, à l'exemple de Price-Mars. Au lieu d'aller à Moscou recevoir une formation d'apparatchik chez Staline, il s'est inscrit à la Sorbonne, à la Faculté d'ethnologie et au musée de l'Homme, où il a suivi l'enseignement de Paul Rivet. Il y a chez lui d'abord une volonté de connaissance. Alors qu'il était un passionné, qu'il venait de créer un parti communiste, qu'il avait été expulsé et emprisonné deux ans pour avoir fondé un parti d'extrême-gauche, maltraité en plein prétoire par la soldatesque de Vincent, il part à Paris. Normalement, pour tous les dirigeants communistes qui avaient fondé un parti [dans l'hémisphère sud de l'Amérique] en Amérique du Sud, et qui ensuite allaient en Europe, le rendez-vous rituel, c'était d'aller à Moscou, où il y avait une école supérieure du Comité central du Parti qu'avait créée le Komintern, où on recevait un " coffre théorique ». Cela voulait dire ce que ça voulait dire, à savoir qu'on était endoctriné essentiellement dans le stalinisme et sa théorie de la Révolution d'octobre. Roumain ne l'a pas fait, alors qu'il pouvait le faire. Alors qu'il avait déjà publié des travaux littéraires de valeur en Haïti, notamment des contes et des nouvelles, il est allé humblement à la Sorbonne, chez le professeur Rivet, au musée de l'Homme, parce qu'il pensait que l'anthropologie était un outil absolument indispensable pour un Noir, pour quelqu'un forgé dans cette pseudo-identité «raciale», afin d'être éclairé : l'anthropologie était là et se forgeait des instruments aptes à contester ses propres théories. Je ne mets pas en question l'anthropologie comme science, mais ses tâtonnements du début, les tâtonnements qu'il y a eu dans une anthropologie un peu fantaisiste, fantasmatique parfois...

Il est intéressant de noter que les Haïtiens se sont intéressés à l'anthropologie parce qu'ils ont eu le sentiment que c'était la discipline qui s'était occupée d'eux. Ils ont voulu voir de plus près. Je n'étais pas du tout, en ce qui me concerne, préparé à lire des ouvrages d'anthropologie, pourtant Dieu sait si j'en ai lu par la suite, à l'exemple de Price-Mars qui était médecin, de Jacques Roumain qui était un homme de lettres... Nous nous sommes dit que c'était la branche du savoir qui, depuis le XviII ${ }^{e}$ siècle, s'était occupée de nous, de qui nous étions, d'où nous venions, qui nous avait appelés "Noirs», qui avait décidé de mettre un nom générique sur l'ensemble des ethnies très différentes qui étaient importées sur les bateaux négriers qui venaient d'Afrique, des gens qui étaient aussi étrangers les uns aux autres que peuvent l'être un Scandinave, un Espagnol, ou un Croate... Malgré la diversité des cultures, des religions et des peuples en Afrique, il avait été décidé que tout le monde était noir. Cela nous a intrigués. Les premiers intellectuels ont voulu se pencher sur les sources de ce savoir. Et certains se sont égarés comme 
Duvalier dans cette expérience. Les Noirs Américains ont fait de même: un homme comme Du Bois s'est intéressé beaucoup à l'anthropologie, C. I. Robert James aussi, en historien de Trinidad.

Le premier geste de Roumain, dès son retour en Haïti, n'a pas été de reprendre le vieux parti communiste qu'il avait laissé, et qui avait disparu en son absence, mais de créer un Bureau d'ethnologie avec Price-Mars. Cela montre nettement qu'il était un intellectuel discipliné et méthodique. D'un autre côté, on trouve pendant la guerre dans sa correspondance avec sa femme Nicole, l'éloge de Staline et des attaques violentes contre Trotski... Mais ce n'est pas cela qui l'obsédait parce qu'il s'occupait d'anthropologie et comptait se lancer dans ses premières recherches. Certains disent qu'il n'a pas été un grand anthropologue, c'est vrai. Ce n'était pas son champ d'action. Il a très peu travaillé en anthropologie. Il est rentré en Haïti au début de 1941, il est mort en 1944. Je ne vois pas comment peut se former une réputation d'anthropologue en si peu de temps dans un pays où l'anthropologie n'existait pas, à part les incursions qu'avait faites Anténor Firmin et Price-Mars. Le travail de Roumain est un travail littéraire, un travail de poète, un travail d'écrivain. Même s'il n'avait écrit que Gouverneurs de la rosée, il serait resté un grand auteur dans les lettres francophones, dans la culture française et haïtienne.

C'était un homme séduisant. Il appartenait à un monde " patricien » tout en prenant ses distances. Il est certain que son statut était assez ambigu, parce qu'il aimait fréquenter le peuple, il allait à la campagne, il faisait ses enquêtes avec Métraux, il retrouvait les paysans de son enfance. Je l'ai connu dans des circonstances particulières comme je le raconte dans la préface que j'ai écrite pour ses CEuvres complètes. Il m'a ramassé alors que je faisais de l'auto-stop sur la route de Pétionville ; il m'a amené chez lui, il m'a offert des livres de sa bibliothèque... Je l'ai vu une seule fois de ma vie, mais il m'a fasciné. J'en garde un souvenir d'émerveillement, parce que c'est la première fois que j'ai rencontré physiquement - je ne connaissais pas encore Price-Mars - un membre de l'intelligentsia haïtienne, et c'était ni plus ni moins Jacques Roumain! Pour nous, c'était une légende à cause de son séjour en prison. Mes oncles, quand j'étais enfant, en 1937, parlaient de lui en termes dithyrambiques.

[...]

Lorsqu'il a commencé à me parler, j'ai découvert un gentleman, un homme très fin, qui avait à mon endroit, alors que j'étais un pauvre garçon, des égards, une gentillesse extrême. Il m'a amené chez lui : jamais je n'étais rentré dans un foyer de la haute bourgeoisie haïtienne, et c'était la première fois que je voyais une bibliothèque de ma vie. J'avais certes déjà vu des livres, éparpillés, dans certaines maisons. Cette fois-là, il m'a fait entrer dans une salle où il n'y avait que des livres... Une bibliothèque avec une échelle ! J'étais fasciné. J'entrais chez un homme de culture, un uomo di cultura, comme on le dit de manière plus forte en italien... Il m'a fait lire Faulkner, Hemingway, Barbusse, il m'a parlé de Roger Martin du Gard, de Malraux, et même de Mauriac, et de Saint-Exupéry, parce qu'il était très ouvert. Il avait dans sa bibliothèque toutes sortes d'auteurs, comme Bernanos que j'ai lu chez lui. J'étais fasciné. Je ne suis pas devenu marxiste, parce que, à l'époque, j'étais chrétien (à cette époque, je fréquentais en même temps les pères du Saint-Esprit, qui me donnaient mes leçons de latin et je lisais Roumain...). J'ai vécu pendant un an une époque mixte et complexe où, comme adolescent, je me cherchais, puis j'ai fini par tomber sur les livres marxistes qui m'ont rapproché brusquement de Jacques Roumain. 
21 Sa mort a été pour nous un désastre, parce qu'il n'y avait personne autour de lui capable de reprendre l'idée du parti, parce que nous, les jeunes dans la clandestinité, on n'était pas un parti mais un cercle de l'opposition à Lescot. À partir de la fin de l'année 1944 et du début de 1945, on a constitué un petit groupe révolutionnaire. On faisait nos premières lectures marxistes, on se réclamait de Roumain. Ce n'était pas un parti communiste, c'étaient des jeunes gens comme on en trouvait à la fin $\mathrm{du}_{\mathrm{XIX}} \mathrm{e}^{\mathrm{e}}$ siècle, des jeunes inquiets qui voulaient faire de l'action sociale, qui voulaient intervenir sur la scène, qui se sentaient des qualités d'hommes d'action. On a lu les premiers textes de Lénine et de fil en aiguille, on a lu Staline, on a lu Marx, on a lu Engels, mais d'une façon très désordonnée, sans aucune méthode, sans rien. Et on a fait la jonction avec les amis de Roumain après sa mort. On a connu Étienne Charlier qui était docteur en droit de l'Université de Paris, Antony Lespès qui était un homme de culture. Aussi invraisemblable que cela puisse paraître, malgré l'analphabétisme qui règne en Haïti, on pouvait côtoyer des hommes de grande culture. Certains d'entre eux étaient des autodidactes, des hommes de la Renaissance égarés en Haïti... J'ai connu Thomas Lechaux qui n'avait jamais quitté Haïti et qui avait des ambitions de tout connaître et connaissait tout de la culture universelle. J'ai croisé des Haïtiens de cet acabit, comme Max Hudicourt ; c'est un type humain (on peut même dire anthropologique...) que l'intellectuel haïtien... On a en effet beaucoup mis l'accent, depuis Anténor Firmin, sur l'importance pour le Noir d'être fort, d'être cultivé pour ressembler au Blanc, d'être capable de parler, capable d'éloquence dans une assemblée, pour pouvoir convaincre, et pour affirmer la valeur de l'individu. Il y a donc eu des gens qui se sont perfectionnés, qui ont étudié, qui ont appris des langues. Il y a eu des gens qui parlaient allemand sans avoir jamais quitté Haïti, qui connaissaient Goethe... Je connaissais un type qui s'appelait Bibine Dupuis qui me récitait tout Faust en allemand! Roumain avait d'ailleurs appris l'allemand pour pouvoir lire les écrits marxistes dans le texte.

Ce sont des choses qui en même temps témoignent qu'il y a en Haïti une valeur humaine qui se cherche, qui ne s'est pas du tout trouvée en politique et qui s'est rabattue sur la peinture, sur la musique... Il y a un imaginaire haïtien d'une grande richesse. Alejo Carpentier m'a ouvert un jour l'esprit à ce sujet (il a beaucoup réfléchi à cela dans ses pérégrinations en Amérique du Sud) : vous traversez l'Atlantique sur un bateau négrier, avec des gens que vous ne connaissez pas, qui sont d'une autre couleur de peau, qui vous ont mis sur le bateau et qui vous ont forcé à une traversée mouvementée, dans des conditions sanitaires épouvantables, au travers des tempêtes et de circonstances climatiques éprouvantes... Puis vous débarquez sur des îles paradisiaques, vous voyez des gens que vous n'avez jamais vus, vous découvrez les Indiens, une autre catégorie d'hommes et de femmes, des arbres que vous ne connaissez pas... Tout cela a élargi l'échelle de l'imaginaire chez les premiers esclaves, chez ceux qui ont vécu cette expérience, avec un système de travail, des outils, des animaux nouveaux. Ils étaient jetés dans un monde tellement différent de celui qu'ils avaient connu sur le continent africain, que cela ne pouvait qu'agrandir l'échelle de perception de soi et de la nature. Et c'est ce qui a débouché sur le réel merveilleux en littérature. C'est de là que provient le réel merveilleux, qui est - et c'est pour cela que Carpentier emploie le mot « réél » - le fruit d'une expérience réelle des premiers temps de la plantation, qui a marqué les imaginaires, et a fait que le vodou est devenu une religion tellement débridée, tellement pleine de fantaisies surréalistes. Au Brésil, il s'est passé la même chose. 
23

En Haïti, tout cela a été concentré dans un univers qui avait réussi économiquement, dans le cadre d'un monde colonial finalement très cohérent. Il commençait, au Cap-Français, à se constituer une société sur le mode parisien. Il y avait des fêtes, toute une société qui se construisait au moment de la Révolution française, de ce fait les gens ont acquis une forme de vision très originale qu'on a retrouvée plus tard dans la peinture haïtienne, dans son sens de la couleur. Ce sont des gens absolument analphabètes qui ont peint, avec le talent d'agencer des couleurs, pour offrir une vision sympathique et généreuse de la nature et $\mathrm{du}$ monde, pour exprimer leur espoir comme de grands peintres. Dans la littérature, c'est pareil. Depuis le XIX ${ }^{\mathrm{e}}$ siècle, on l'a vu chez un poète comme Oswald Durand qu'on a oublié, qui était un des premiers poètes haïtiens, influencé par le symbolisme. Et chez des gens comme Léon Laleau, avant Roumain, qui ont été de bons poètes. Comme chez Magloire Saint-Aude, Dany Laferrière, Emile Ollivier, Frankétienne ou Davertige (Villard Denis)...

24

Je pose la question : que peut représenter Roumain, aujourd'hui, pour Haïti? Que peut apporter un homme d'une telle sensibilité, d'un tel talent? D'abord, Gouverneurs de la rosée, plus que jamais, est un livre que tout Haïtien devrait relire en ces temps, parce qu'il nous replonge dans la partie la plus authentique de nous-mêmes, et dans la tragédie de l'eau en Haïti. À tous les points de vue, on trouve chez Roumain un imaginaire qui nous réconforte dans l'estime de nous-mêmes, dans l'idée qu'ont les meilleurs Haïtiens de changer la vie du pays. Roumain était un homme qui voulait changer Haïti de fond en comble, qui luttait pour cela. Son message, si sans doute il y a un message de Roumain, c'est un message d'espoir. Je pense qu'il faut enseigner Roumain à l'école. Il faut d'abord créer un enseignement et en plus traduire tout Roumain en créole. C'est la première chose à faire du point de vue pédagogique pour qu'il soit connu. Je crois que c'est la plus grande école d'espoir que nous ayons dans la littérature haïtienne. On néglige souvent sa poésie parce que c'est une poésie d'une grande violence, une poésie où figurent les rares poèmes d'inspiration internationaliste qu'on possède. Les poèmes de Bois d'ébène sont des poèmes d'inspiration prolétarienne, si on peut dire. Il y en a très peu. Il n'y a que Maïakovski et Césaire qui aient atteint cette force lyrique qu'a Roumain dans ses poèmes anticolonialistes...

Roumain aura été l'un des rares poètes noirs à se démarquer tout en s'appuyant - et là il montre la dialectique de la vie - sur ce qu'on nous a imposé. Puisque nous avons vécu pendant des siècles sur l'idée que nous étions des Noirs et que nous en avons fait notre miel, notre danse, notre vision du monde, on ne peut pas la rejeter purement et simplement. Nous avons vécu trop longtemps avec cette conscience malheureuse pour la rejeter sans autre forme de procès. Mais il faut la dépasser. Et c'est ce que Roumain fait dans les poèmes de Bois d'ébène, qui ont une portée universelle où on voit un homme surmonter ses contradictions et les rendre fraternelles, les rendre fécondes. C'est un grand mérite.

26 Cela gêne beaucoup de gens: il y a une tendance même à occulter Roumain. J'ai découvert, à ce propos, qu'il y a une sorte de réticence vis-à-vis de Roumain, à cause de la violence de ses poèmes, à cause du fait qu'il a été, même dans la direction prolétarienne, au-delà des malheurs que le communisme a causés au prolétariat mondial. Dans Gouverneurs de la rosée, ce n'est pas du réalisme socialiste: il a transcendé le réalisme 
socialiste, il en a fait une œuvre d'art tout court. Et je pense qu'ils sont très peu nombreux, parmi les poètes qui ont été communistes, qui y ont réussi : sans doute Maïakovski, Césaire, sans doute Aragon, quoi qu'on dise, Paul Éluard, Raphaël Alberti, Pablo Neruda, Cesar Vallejo, Yannis Ritsos, Guillevic, et même quelqu'un qui n'était pas communiste, Kazantzakis, mais qui était très proche, ou bien Cesare Pavese, en Italie. C'est valable pour Bertold Brecht, pour le poète tchèque Vitezslav Nezval, pour certains poètes macédoniens que j'ai lus, pour l'Allemand Stefan Hermlin, pour Nicolas Vaptzarov, un poète bulgare. Il $\mathrm{y}$ a aussi un certain nombre de poètes du mouvement révolutionnaire, en Amérique latine notamment. Nicolas Guillén, qu'il ne faut pas oublier, Langston Hugues aux États-Unis et d'autres poètes révolutionnaires latino-américains qui - je ne dis pas qu'ils réhabilitent le marxisme - montrent qu'il n'y avait pas eu que des slogans meurtriers dans le mouvement communiste international. Il y a eu des œuvres d'art authentiques. On ne peut pas jeter à la poubelle purement et simplement tout ce qui a été marqué par l'expérience de la Révolution d'octobre. Il y a eu des choses magnifiques parce qu'elles étaient vécues dans l'authenticité. Roumain est un homme qui a vécu dans l'authenticité, qui a vécu le marxisme comme une aventure spirituelle et esthétique, pas seulement comme une pratique politique, comme une stratégie révolutionnaire. Plus que cela, il l'a sublimé dans des œuvres de valeur, ses nouvelles, ses contes, ses poèmes, ses essais.

[...]

Il y avait dans l'entourage de Price-Mars un autre courant, un courant obscurantiste, issu lui aussi de la Revue Indigène, qui s'était constitué parallèlement autour de la revue Les Griots, avec les trois D : François Duvalier, Lorimel Denis et Louis Diaquoi. Ils étaient toute une équipe d'intellectuels noirs, qui, frustrés dans leur vie sociale, souffraient de n'être pas admis dans certains clubs mondains de la capitale où on voyait les plus belles filles de Port-au-Prince. Du fait de leur couleur, ils étaient réellement victimes d'un ostracisme. Sous Lescot, cela s'était aggravé. Lescot avait créé un favoritisme très marqué à l'égard des mulâtres. C'était manifeste dans les clubs, dans les familles, dans les fêtes... Ces jeunes gens, dans les années 1930, sous Vincent, avaient déjà beaucoup souffert. Ils se réclamaient aussi de Price-Mars, mais ils n'en avaient retenu que le côté africaniste. Ils pensaient que Price-Mars célébrait l'Afrique alors qu'il faisait plutôt la lumière autour des origines africaines de la culture haïtienne, et qu'il tenait compte du fait qu'il y avait plusieurs composantes dans la culture haitienne, une composante française qui se manifeste par exemple dans la langue que Price-Mars maniait très bien. Il n'avait pas honte de ses études à la Faculté de médecine de Paris. Il s'en réclamait. Mais eux n'ont retenu que le "noirisme». Ils ont voulu faire une sorte de racisme antiraciste. Ils se seraient arrêtés à une sorte de "négrisme", mais comme le terme était connoté directement « Amérique du Sud » - on ne disait pas négrisme en Haïti -, ils ont employé le terme de "noirisme ». C'était une « doctrine » véritablement raciste, constituée autour de manifestations raciales, de sentiments raciaux...

Après la mort de Roumain, ils ont pris possession du Bureau d'ethnologie. Price-Mars luimême n'avait pas le temps, et ce sont eux, Lorimer Denis surtout, qui l'ont dirigé. Ils en ont fait une officine raciste pour préparer la prise du pouvoir par François Duvalier. Ils ont formé tout un tas d'épigones très médiocres. Ce qui m'a frappé chez ces gens, c'est leur médiocrité intellectuelle, y compris chez Piquion. Ils étaient nuls du point de vue de la science sociale. Le Bureau d'ethnologie est donc tombé entre les mains d'un groupe 
d'extrême-droite que j'ai connu, qui appartenait à ma génération. Parmi eux, il y en avait un seul qu'on pouvait considérer comme un véritable ethnologue, Emmanuel C. Paul, qui a fait des études scientifiques de terrain, qui a étudié le folklore, la musique, les contes, mais d'une façon scientifique. C'est le seul qui ait compté dans cette équipe. Je l'ai connu, je l'ai retrouvé à Paris: il se méfiait d'ailleurs de Duvalier et des autres... Dans nos conversations personnelles, il me disait qu'il n'avait rien à voir avec eux, que ce n'étaient pas des hommes de science. Il y en a un autre aussi à qui je devrais rendre hommage, Roger Mortel, qui avait fait un doctorat d'anthropologie à Paris, qui est rentré juste au début de 1958, en même temps que moi, et qui est vite reparti, qui m'a dit: «Je ne reste pas, parce que sinon je vais être intégré. Il n'y aura pas moyen, étant donné que je suis docteur en ethnologie de la Sorbonne, de sortir de cette équipe.» On l'aurait assassiné. Il a compris à qui il avait affaire parce qu'il avait accès à Duvalier, il l'avait entendu ; il avait vu quel esprit fumeux et intolérant, voire terroriste, c'était.

Duvalier-Papa Doc a travesti l'ethnologie purement et simplement. Ce qu'il a retenu de l'ethnologie, c'est un retour à une Afrique mythique de son cru, non pas à l'Afrique comme nous la connaissons, à l'Afrique qui a été colonisée, aux sociétés africaines qui luttaient à travers des syndicats, des mouvements comme le panafricanisme, notamment dans l'Afrique de langue anglaise, ou comme le Rassemblement démocratique africain (RDA) autour de Senghor, Sekou Touré, de Houphouët-Boigny... Tout ce mouvement de décolonisation, il l'a ignoré pratiquement. Ce qui l'intéressait, c'était l'Afrique éternelle, l'Afrique-mère. Il a créé toute une mythologie de pacotille autour de l'Afrique, une idée très duvaliérienne de l'Afrique qui ne correspondait pas à la réalité.

Une rhétorique vide..., vide, vide, qui ne connaissait pas les progrès de l'ethnographie, Duvalier n'avait pas lu Delafosse, il n'avait pas lu Michel Leiris, il ne s'intéressait pas aux disciplines universitaires... Pour lui, ethnologie et noirisme revenaient au même, selon une "doctrine " qu'on pourrait appeler aujourd'hui la "papadocratie ", très fumeuse, très abracadabrante, très obscurantiste. Ses « œuvres essentielles », plusieurs volumes de ses œuvres complètes, qu'il appelle lui-même des œuvres essentielles, sont illisibles. Je l'ai bien connu personnellement; j'ai eu des conversations approfondies avec lui, des discussions, des débats. Il passait du coq à l'âne, c'était un sottisier ambulant... Si je rassemble les propos que tenait Duvalier - je le ferai peut-être dans mes mémoires -, c'était sans queue ni tête. C'était quelque chose d'affreux, d'insupportable. C'était pénible de parler avec lui. On avait honte d'entendre quelqu'un dire autant de stupidités en une heure de temps! J'ai fini par rompre avec lui, et à mon retour en Haïti, en 1957, j'ai compris que c'était un homme dangereux parce qu'il a rattaché aussi à cela tout un nationalisme de bas étage. Il n'a retenu que très peu de chose de Mustapha Kemal, qu'il admirait beaucoup, mais du kémalisme, il n'a rien gardé, ni même de Salazar. Il faisait un mélange entre Mustapha Kemal, Salazar, Tchang Kaï-Chek et plus tard Franco. Au fond, c'était ce que Leslie Manigat devait appeler « le fascisme de sous-développement ». C'était un fasciste, un fasciste tropical, contemporain de Franco, que lui et Piquion au fond admiraient profondément, en hommes d'extrême-droite.

Voilà Duvalier. Je ne noircis pas le bonhomme. Je l'ai bien connu. J'avais d'abord fait sa connaissance en tant qu'un charmant médecin de quartier qui souvent nous avait guéris du paludisme. Mais après, je l'ai perdu de vue pendant onze ans, durée de mon premier séjour à l'étranger. Quand je suis rentré, je me suis trouvé en face d'un dictateur de la pire espèce, comme il l'a montré. C'était en fait un pauvre homme, un minable, mais 
redoutable, un tyran criminel... Je combats absolument l'idée qu'on a eu affaire à un ethnologue avec lui : un ethnologue ne devient pas un assassin, un homme de sac et de corde! Des gens comme Piquion ont été plus que complaisants vis-à-vis de lui. Piquion a profité des prébendes du dictateur pendant des années, et jusqu'à sa mort, il est resté un duvaliériste bon teint. Le malheur pour Duvalier, mais ce fut un bonheur pour nous, c'est que, en dehors d'Emmanuel C. Paul, il n'a pas eu, autour de lui, de personnes de talent, alors qu'Haïti était bourrée de gens de talent.

Revue « Les Griots »

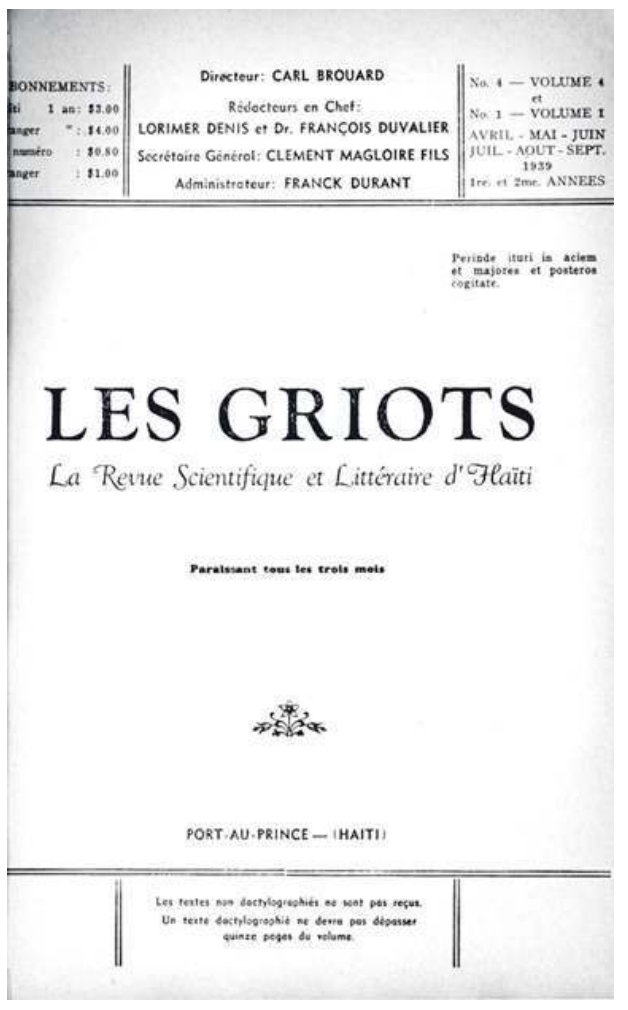

droits réservés

$[\ldots]$

Jacques Stephen Alexis, je l'ai connu très tôt, un peu à la même époque où j'ai connu Roumain, quand nous avons fait notre petit journal La Ruche. [...] Il était à la faculté de médecine quand j'étudiais encore pour le baccalauréat. Quand nous avons fait notre journal, Théodore Baker et moi, nous avons mis Alexis et Gérald Bloncourt sur la liste des éventuels collaborateurs, et ils ont accepté de se joindre à nous... Dès le premier numéro, on s'est démarqué de la presse haïtienne habituelle. Un ton de jeunes gens qui débarquent avec fracas dans la vie, une violence verbale sans précédent en Haïti pour attaquer toutes les institutions, l'Église, l'armée, la famille, à la manière surréaliste, iconoclaste. Un succès fou... Les gens s'arrachaient le journal. On a dû augmenter le tirage. Alexis écrivait une chronique qui s'appelait «Lettre aux hommes vieux", qui était très brillante... Troisième numéro. Breton arrive en Haïti, invité par Mabille... Nous réunissons notre petit comité de rédaction. Qu'est-ce qu'on va faire? Il faut mettre le paquet: on n'aura pas tous les jours quelqu'un de la classe de Breton présent dans le pays... Que mettre en manchette? J'ai dit que je ne voyais qu'un seul titre, "Vive l'insurrection nationale!» Dans un pays comme Haïti, c'est comme s'asseoir sur un baril de poudre! Le journal 
paraît, bien présenté, tout entier consacré au surréalisme, avec un hommage à André Breton, mais avec des titres incendiaires.

Vous imaginez? "Ils" n'ont pas aimé. Baker et moi, nous avons été arrêtés, naturellement... Le bruit court dans la ville que nous sommes en prison, ce qui déclenche de grandes manifestations devant le palais pour demander notre libération. La police charge. Nous sommes les 7, 8, et 9 janvier 1946. L'armée, qui ne voulait pas que les choses dégénèrent, est alors intervenue à l'insu du gouvernement. Elle a fini par arrêter le président et par nous donner raison. Nous sortons dans la rue, en criant victoire. Je monte à l'étage du quartier général de l'armée, je fais un tabac en haranguant 40000 personnes! Et pendant deux ou trois jours, les 11, 12 et 13 janvier, nous sommes maîtres de l'asphalte, maitres du terrain. Pendant trois jours, il y a un vide constitutionnel. Alexis a 23 ans, j'ai 20 ans, mais on n'arrive pas à prendre le pouvoir, parce qu'on est trop jeune. L'armée forme un comité exécutif militaire et nous apprend que désormais il faut compter avec elle, qu'elle constitue le pouvoir et nous demande quelles sont nos intentions. Nous maintenons l'agitation pendant une semaine, mais l'armée nous dit que cela suffit, que le gouvernement dictatorial a été renversé, que tout doit rentrer dans l'ordre, et qu'il faut regagner nos familles, comme ils disent. On répond aux militaires qu'on ne rentre nulle part, qu'on veut la démocratie et que nous allons fonder tout de suite le parti communiste.

Alexis, Baker, Bloncourt et moi, nous sommes devenus «célèbres » en Haïti. Tout le monde avait nos noms sur les lèvres, nous étions des leaders. Nous avons failli prendre le pouvoir, mais nous avons été très vite isolés, écartés, vivement attaqués par la presse traditionnelle. À cause de nos idées on nous a traités d'extrémistes, d'anarchistes, de libertins et nous avons été obligés de quitter le pays parce que notre vie aurait pu être menacée. Les militaires ont pris le pouvoir, un gouvernement a été élu pendant l'été 1946, celui de Dumarsais Estimé. Dès octobre, on sentait qu'on faisait tout pour que nous quittions Haïti...

Avant de partir pour Paris, on a jeté quelques bases avec nos amis qui ont pris en main le parti communiste, qui à l'époque était légal, mais devait être condamné à l'illégalité peu de temps après. Alexis est allé terminer ses études médicales, en se spécialisant en neurologie à la Salpêtrière, avec le professeur français Alajouanine. Moi, je me suis inscrit à la Sorbonne et à Sciences Po. Alexis devait ensuite faire une œuvre littéraire considérable; il devait d'abord publier un roman qui a eu un grand retentissement; il s'appelle Compère Général Soleil. Alexis a, finalement, peu à peu laissé de côté la médecine pour uniquement écrire des romans. Il a été très bien accueilli en France, la critique a été unanime, notamment pour Compère Général Soleil, publié chez Gallimard. On peut dire que, après Roumain, on n'a jamais eu un écrivain de cette importance, qui promettait tant... Alexis me montrait des synopsis de ses livres. Il avait plus d'une quarantaine de titres en attente. Il allait être un écrivain extrêmement fécond, parce qu'il écrivait vite et il avait déjà le tracé d'une œuvre abondante de romancier. Il connaissait bien Haïti, il avait énormément de talent et c'était un homme extrêmement studieux. J'ai rarement vu quelqu'un d'aussi studieux. J'ai raconté, en préface à un de ses livres, à quel point il s'intéressait à tout. C'est un peu le trait de ma génération, à cause de l'influence de Jacques Roumain, qui connaissait aussi bien l'anthropologie, la poésie, l'économie, la littérature... On voulait suivre son exemple, Alexis particulièrement, on lisait tout. Comme il avait une formation scientifique, il lisait aussi les hommes de science. Il est 
rentré en Haïti avant moi. Il a pris des contacts, il s'est lié profondément à l'intelligentsia haïtienne, aux gens de théâtre.

À mon retour en Haïti, en 1957, j'ai constaté qu'il existait une division dans le groupe révolutionnaire haïtien. Il y avait deux partis. Un Parti des démocrates populaires et un autre parti, à l'état de noyau, qui allait devenir le Parti de l'entente populaire. Alexis et moi on ne se retrouve pas dans le même parti... Alors que je m'installais à Cuba, après un nouveau départ d'Haïti, Alexis est allé à Moscou, où il a fait reconnaître son parti par le parti communiste d'Union soviétique, par Khrouchtchev, dont il est devenu l'ami, après avoir fait une intervention très brillante, très remarquée à la conférence des 81 partis communistes qui a eu lieu en 1960. Quelque temps après, Alexis disparaît. On s'inquiète, le parti communiste cubain fait des démarches. Un beau jour, après des mois, fin 1961, je suis convoqué à la direction du parti cubain et on me dit qu'Alexis aurait été assassiné, après avoir débarqué en Haïti avec un groupe de jeunes gens partis de Cuba. Alexis ne m'avait pas dit ce qu'il préparait. Je ne lui en veux pas parce que je pense que ce sont des choses secrètes qu'il n'avait pas à me dire, que son groupe préparait, non pas un débarquement, car ils n'étaient pas armés, mais une infiltration en Haïti. On me dit qu'il avait une grosse somme d'argent sur lui (Khrouchtchev lui avait remis une somme énorme, quelque chose comme 45000 dollars...). Maintenant, je connais toute l'affaire, mais on a mis du temps à savoir ce que je vous dis là. Le groupe a été intercepté dans une partie du Môle Saint-Nicolas qui s'appelle Bombardopolis, une bourgade du nord d'Haïti, près de Port-de-Paix. [...] Cette bourgade dont parle Dany Laferrière dans son roman Pays sans chapeau, où les gens n'ont plus besoin de s'alimenter, au grand étonnement des scientifiques.

\section{[...]}

Alexis a été massacré, sacrifié. Il parait qu'on l'a torturé, jeté à la mer. Les services secrets cubains l'ont appris bribe par bribe et m'ont communiqué peu à peu, avec les années, les informations. C'est comme cela qu'Alexis a disparu. On ne peut pas dire que c'est la division du parti qui a été la cause de son échec, pas du tout. Il s'est lancé dans une action audacieuse, mais il n'a pas attaqué avec des armes. S'il m'avait dit cela, je n'aurais pas été d'accord parce que j'aurais voulu qu'on fasse - j'avais déjà reçu une petite formation militaire à Cuba à ce moment-là, que le Che nous avait donnée - un entrainement de guérilla. Mais Alexis avait l'intention de débarquer en s'infiltrant avec de l'argent pour travailler de l'intérieur. Ce n'était pas absurde de faire cela, mais il faut dans ce cas avoir une logistique sur place qui vous accueille, qui vous reçoit. Et il n'y en avait pas. Quand ils ont débarqué, personne ne les attendait, des curieux les ont vus, les ont dénoncés aux tontons macoutes du coin. Ils ont été isolés, interceptés, capturés, tués avant même que Port-au-Prince soit consulté, parait-il. C'est parce que les tontons macoutes ont découvert l'argent qu'Alexis portait qu'ils les ont assassinés pour prendre le fric. C'est là une vie absolument douloureuse qui s'est terminée par un calvaire, avec des jeunes gens qui ont aussi tous péri.

Alexis reste dans les mémoires des jeunes, de tout le monde en Haïti, comme un des héros de la lutte pour la libération d'Haïti parce qu'il a été victime de la barbarie du duvaliérisme, et le fidèle continuateur de Jacques Roumain. Il a écrit une œuvre originale qui ne ressemble à aucune autre, qui ne ressemble pas aux œuvres des premiers romanciers Marcelin et Hibbert, qui n'imite pas Jacques Roumain, une œuvre qu'Alexis seul pouvait produire, avec des textes de grande valeur comme Les Arbres musiciens, 
Romancero aux étoiles et surtout Compère Général Soleil. C'était assurément un grand écrivain.

Comme Roumain, il appartenait à la meilleure société [...]. Son père Stephen Alexis était un ancien diplomate. Il avait été ambassadeur en Belgique, à Londres. C'était un homme de grande culture, que j'ai connu jusqu'à sa mort, car il est mort longtemps après son fils en exil à Caracas (j'étais en correspondance avec lui). C'étaient des gens d'une grande originalité, des Noirs hors du commun, le père et le fils. Je n'ai jamais connu des hommes aussi originaux. Quand j'ai écrit sur Jacques, mon texte n'a pas plu à ses amis. Ce qui fait qu'aujourd'hui des gens ont cru que nous étions des ennemis, des adversaires, alors que j'ai simplement voulu montrer la grande complexité d'Alexis. Je l'ai connu comme un frère. On a vécu ensemble dans la même chambre d'étudiant à Paris... Il avait un côté mythomane, dans le meilleur sens du mot...

[Et Aimé Césaire... ?] Avant notre aventure révolutionnaire de 1946, Césaire nous avait, un an auparavant, mis au courant de ce qu'était le surréalisme: nous avions suivi son séminaire pendant sept mois en 1944. C'est Césaire qui est à l'origine de notre ébullition. Nous avions Roumain, mais Césaire a ajouté à notre ignorance du sang neuf: la haute parole surréaliste de Lautréamont, de Rimbaud...

Césaire est resté sept mois en Haïti. Il ne faut jamais l'oublier. Et c'est là qu'il a acquis sa connaissance d'Haïti.

Il était aux obsèques de Roumain. Il était professeur à la faculté mais, comme il était Français, il faisait attention à ne pas se mêler de politique. Il faisait son cours, mais son cours était incendiaire... Et le gouvernement ne le savait pas. Un Noir français qui enseigne, un Martiniquais de haut vol... On ne donnait pas d'importance à un Noir Martiniquais qui enseignait. Césaire nous a donc portés sur les fonts baptismaux, en même temps que Roumain et Price-Mars. Il ne faut pas l'oublier. [...] J'ai toujours été très attaché à Césaire, même quand j'avais des réserves sur la "négritude " parce que je craignais (et mes craintes étaient fondées) qu'en partant d'une idéologie qui met l'accent sur la race, qui réaffirme la valeur esthétique de la race noire, qui reprend, mais en les inversant, les termes de l'anthropologie classique qu'avait créés le Blanc, il y ait un risque qu'un aventurier s'empare de ce concept et en fasse un intégrisme, un fondamentalisme comme on pourrait dire aujourd'hui. J'avais parlé à l'époque d'un «sionisme noir » tout en ayant du respect pour les sionistes d'Israël, mais Césaire avait très bien compris cela.

Je me méfiais de l'utilisation d'un mot comme «négritude », issu du terme «nègre » qui appartenait à l'anthropologie : même si on l'amenait sur le terrain esthétique, si on en faisait une plate-forme esthétique pure et simple, il continuait à avoir une connotation raciale. Quand on dit "négritude », on a l'impression qu'il renvoie à la « race » noire. J'ai clarifié ma position vis-à-vis de Senghor, et vis-à-vis de Césaire. Les craintes que j'avais étaient justifiées parce que, quelques années après, Duvalier devait prendre le pouvoir, sous le couvert d'une sorte de négritude totalitaire. C'était de la négritude dévoyée, qui n'était articulée à aucune œuvre littéraire mais un centre absolument fasciste comme ce qu'était devenu l'Institut d'ethnologie avec Duvalier... Donc je devais me méfier de ce concept et de tout concept forgé à partir d'une théorie raciale. La négritude aura été cependant féconde dans la mesure où elle est à l'origine des œuvres de Césaire, de Senghor, des grands poètes « noirs », de Léon Gontran Damas et des autres.

Gradhiva, 1 | 2005 
44

Ce qui a sans doute conduit Breton à aimer Haïti, à parler d'Haïti, à vouloir faire un séjour en Haïti, c'est qu'il avait été influencé déjà dans sa jeunesse par l'art africain, aux côtés de Picasso et de Braque. Je pense que c'est là qu'il faut chercher. Il a vu la beauté de l'art africain, des masques, dans une voie ouverte par Apollinaire et Cendrars. Il a toujours eu une grande sympathie pour les arts des pays africains, et aussi du Mexique. Quand Mabille a été envoyé en Haïti comme attaché culturel de la France libre, il l'a encouragé à y venir. Breton avait d'autre part rencontré Césaire en 1941 : sa curiosité était stimulée par le fait qu'il avait découvert l'énorme talent de Césaire, le génie de Césaire... Quand il a su que Césaire avait vécu en 1944 en Haïti, il a voulu aller lui-même y faire un tour.

Wifredo Lam, le peintre cubain, s'intéressait aussi à Haïti. Il y a eu toute une série de circonstances - ses amis, son expérience de l'art africain - qui l'ont incité à aller dans un pays comme Haïti. Il a été très surpris de tomber sur Magloire Saint-Aude, qui était un poète surréaliste magnifique et sur des jeunes poètes, dont je faisais partie, sur René Bélance et Paul Laraque qui étaient pleins d'admiration pour lui. Césaire nous avait préparés à recevoir Breton, ce n'était pas pour nous un inconnu. Et sa présence a coïncidé avec un mouvement contestataire, libertaire qui ne pouvait que lui plaire. On lui a fait une fête considérable, au cours d'une soirée extraordinaire qui était une fête surréaliste. Avec Breton, on a pratiqué le surréalisme dans la vie quotidienne, ce qui lui plaisait beaucoup, et qu'il avait connu dans sa jeunesse. Notre rencontre a été un acte surréaliste de premier ordre...

C'est la première fois qu'il arrivait au surréalisme de descendre dans la rue comme il avait rêvé de le faire, pas pour tirer à droite et à gauche sur la foule, mais pour s'incorporer à un mouvement de contestation contre un dictateur haïtien. Breton et Mabille ont été expulsés d'Haïti, ce qui n'a pas déplu à Breton. Les autorités militaires d'Haïti leur ont donné quelques jours pour quitter le pays.

47 


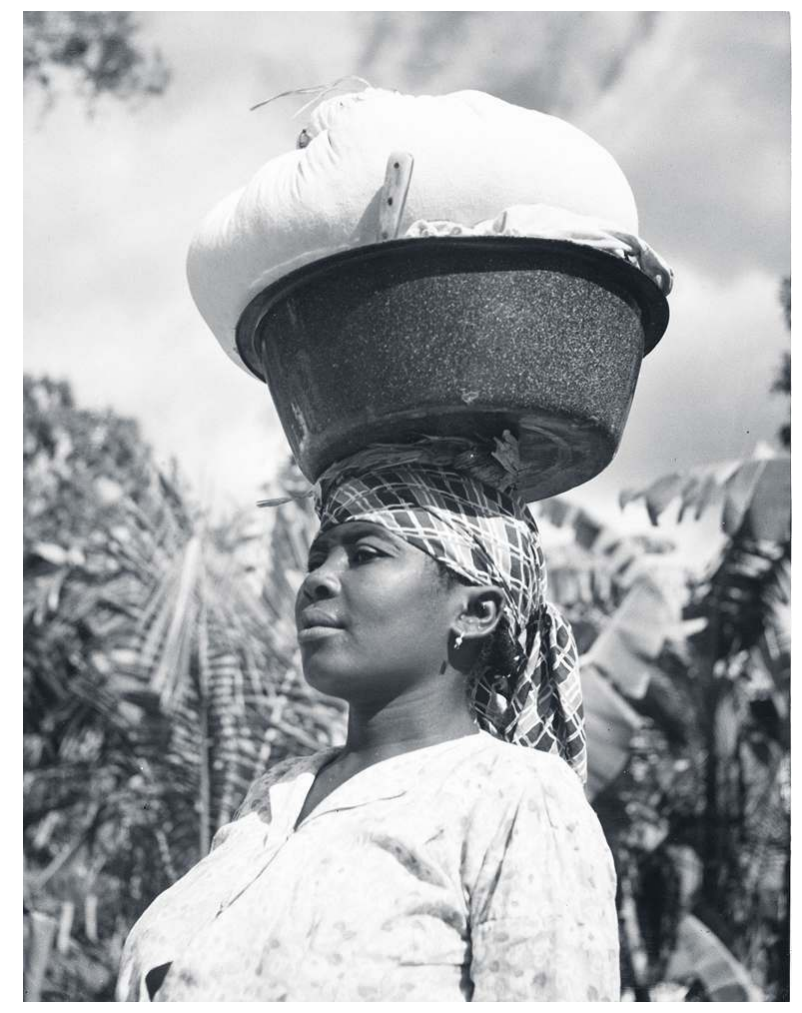

(c) musée du quai Branly, photo Alfred Métraux

J'ai connu Michel Leiris d'abord chez Césaire quand je suis arrivé à Paris en 1946. Le dimanche après-midi il y avait des réunions passionnantes. Césaire m'invitait à participer à des rencontres les dimanches après-midi, au 10 de la rue de l'Odéon. Il y avait là René Ménil, Loeb, Michel Leiris, Jean Cassou, des gens très intéressants, des intellectuels parisiens. C'est comme cela que j'ai découvert Leiris et l'amitié que Leiris portait à Césaire. Beaucoup de choses que je sais de Césaire, c'est de Leiris que je les tiens. Et Leiris m'a toujours manifesté une très grande amitié. Je l'ai rencontré à Cuba quand il est venu avec Lam. Je pense qu'il a fondé l'ethnologie sur des bases nouvelles, je ne sais pas ce qu'en pensent les anthropologues, mais je crois que c'est l'un des anthropologues les plus sérieux.

[Son ouvrage,] Contacts de civilisations, c'est un travail classique d'ethnologie. Je l'ai toujours lu, aussi bien ses livres sur l'Afrique, ses travaux magnifiques sur l'Afrique, et puis ses textes autobiographiques, L'Âge d'homme... Il m'a invité chez lui au quai des Grands-Augustins. Il a beaucoup aimé Haïti. Il est venu à Cuba, on a beaucoup parlé. Je l'ai interviewé pour la radiodiffusion cubaine. J'avais l'honneur d'être un ami de Michel Leiris.

[...]

[À propos des naïfs haïtiens...] Les circonstances font que ma classe de lycée a été à l'origine, du point de vue chronologique, ne serait-ce que cela, du mouvement naïf haïtien. Dewitt Peters, était un jeune professeur d'anglais envoyé dans le cadre de la coopération des États-Unis en Haïti pendant la guerre comme professeur d'anglais au lycée Pétion. Pendant un an, il a tout fait pour nous apprendre l'anglais, je ne crois pas qu'il ait réussi... En tout cas, un beau jour, il est venu et nous a présenté ses adieux parce 
qu'il partait. Quand on lui a demandé s'il rentrait aux États-Unis, il nous a dit non, qu'il allait ouvrir un centre d'art. Il était peintre à ses heures, il allait reprendre la peinture. Mais surtout il nous a expliqué son projet : il allait donner des pinceaux à tous ceux qui veulent peindre comme ils sentent et nous a demandé si parmi nous il y en avait qui voulaient venir le rejoindre : j'ai toujours regretté de ne pas avoir saisi cette occasion cela a été un manque d'intuition - pour comprendre ce qu'il y avait à l'origine de tout cela. Un an après, alors que Breton était en Haïti, il a fait la première exposition d'art des naïfs haïtiens en présence de Wifredo Lam et de Breton. Cette exposition a lancé le mouvement qui devait prendre un essor international à la fin des années 1940 et dans les années 1950. C'est extraordinaire. Je n'ai jamais revu Peters, mais j'étais très proche de son aventure artistique.

51 C'est un critique d'art cubain qui, pour la première fois, a saisi la dimension mondiale de ce mouvement. Il faut dire que Lam nous a beaucoup influencés : il y a eu une exposition de ses œuvres qui a aussi dû ouvrir les horizons des Haïtiens. Lam, la peinture naïve... Cela a créé un lien supplémentaire avec Cuba, en plus de Nicolas Guillén et d'Alejo Carpentier qui étaient des amis de Roumain dans les années 1940.

$52[\ldots]$

[À propos du débat sur la race...] Je pense qu'il y a eu un énorme dérapage de la vieille anthropologie, dont les conséquences ont été plus graves en Haïti qu'ailleurs. Elles n'ont pas été aussi graves en Martinique ou en Guadeloupe, parce que ces pays n'ont jamais été indépendants et ont quand même bénéficié de l'expérience de la laïcité française, du droit français, des pratiques françaises de la vie que nous n'avons pas eues, qui auraient peutêtre permis de pacifier un peu cette violence récurrente qu'on trouve en Haïti. Je crois en effet que ce qui est très grave dans l'histoire de la colonisation, parmi beaucoup d'autres choses, c'est d'avoir ajouté à la traditionnelle, à l'universelle domination de l'homme sur l'homme qu'on connaît depuis le début de l'histoire, une idéologie mythologique comme la notion de " race » pour aggraver la situation matérielle et physique déjà très précaire des gens par une douleur psychologique, car la race n'a engendré que la douleur, la honte de soi, la solitude, une sorte d'incompréhension suicidaire de soi. Je le vois même dans l'expérience Aristide. Aristide est quelqu'un qui s'est suicidé, si on peut dire, parce qu'il avait tous les atouts. Il a perdu, il s'est laissé avoir littéralement pour être obligé de partir, de prendre la fuite au petit matin en gâchant toutes ses possibilités. Il y a une part de suicide dans ce comportement. On peut le voir chez beaucoup de dirigeants haïtiens, dans le comportement personnel de beaucoup d'Haïtiens aussi. Il y a donc une souffrance...

[...]

Indépassable et à vie parce qu'on ne sort pas de la couleur de sa peau. Peut-être une étude montrerait pourquoi en Haïti cette souffrance a pris une telle proportion, pourquoi elle a marqué le développement de l'État, pourquoi elle a contrarié le développement national. Jusqu'à ce jour, l'Haïtien a été généralement obsédé - il y a beaucoup d'exceptions - par cette affaire raciale dans ses rapports avec autrui. Souvent, j'ai remarqué chez beaucoup de mes compatriotes, le genre de rapports qui prédominent quand on est ensemble, mais une fois qu'il y a un Blanc ou plusieurs Blancs, je note le passage à une autre vitesse, à une sorte de qui-vive, à une sorte d'angoisse, à une sorte même de carnaval. Le mot « carnaval » n'est pas venu par hasard à mes lèvres. Je pense que cette affaire raciale a donné lieu à une " carnavalisation » de l'histoire. Depuis 1492, l'Occident n'a pas arrêté de mettre des masques sur des réalités, comme sur les réalités géographiques, en changeant 
les noms. Haïti est devenue Saint-Domingue, après avoir été Hispaniola avec l'autre partie de l'île, l'actuelle République dominicaine... Des masques ont aussi été appliqués aux hommes. Ces indigènes que Christophe Colomb trouve par hasard sur son chemin aux Amériques, qui étaient des Arawaks, des Taïnos, des Caraïbes, il les appelle des « Indiens ", alors que les Indiens se trouvent en Asie, de l'autre côté du monde. La même chose a fonctionné avec les « Noirs » et avec les Chinois qu'on a appelés des « Jaunes »... Je constate le fait: on a masqué pour tromper les gens, pour jouer un jeu machiavélique dans la mainmise politique sur les consciences. Ce n'est pas par hasard que le carnaval, jusqu'à nos jours, a eu une grande importance dans la vie quotidienne des Haïtiens, des Cubains, des Trinidadiens, des Brésiliens...

54 J'ai été confronté au problème racial à Cuba puisqu'avant la révolution, Cuba était le pays le plus raciste de l'Amérique latine. C'est à partir de Cuba que j'ai commencé à vouloir réfléchir sérieusement sur cette problématique raciale. J'avais eu déjà des débats, au moment de la négritude. Je me suis toujours méfié du concept de race, bien que moimême j'aie pensé, et je ne regrette pas de l'avoir fait (je rejoins la position de Sartre par rapport à la négritude), qu'on ne pouvait pas du jour au lendemain se débarrasser, dans notre conscience d'écrivain, de cette affaire raciale en un tour de main, qu'il fallait l'assumer sur le plan de la création elle-même: du point de vue philosophique, il fallait l'utiliser comme une médiation, il fallait en passer par là. C'est comme cela que j'ai interprété le Cahier d'un retour au pays natal de Césaire, la prose et les poèmes de Roumain, les poèmes de Senghor et Damas, et mes propres poèmes de Minerai noir. C'était une expérience à vivre parce qu'on nous l'avait fait vivre, un vécu qu'on nous avait imposé depuis la découverte de l'Amérique.

55 J'arrive à Cuba, dans une société qui a été, beaucoup plus qu'Haïti, mais sous une autre forme, une société à fort antagonisme racial avant la révolution. Je parle avec les Noirs Cubains, je parle avec Guillén, je parle avec tous et je décide d'étudier la question raciale à Cuba. J'ai écrit à l'époque un article dans Présence africaine (1965) en réponse à un jeune Cubain, M. Carlos Moore, qui avait accusé Castro et Che Guevara d'être des racistes. «Le peuple noir a-t-il sa place dans la révolution?", avait-il écrit. J'ai répondu par un long article qui s'appelait « Lettre de Cuba sur l'impérialisme de la mauvaise foi », parce que je trouvais que Moore manifestait dans son article beaucoup de mauvaise foi. Si véridiquement, il y avait du racisme à Cuba, on ne pouvait accuser ni Castro ni Guevara ni le frère de Castro, Raoul, ni l'ensemble du personnel dirigeant de la révolution, d'être une bande de racistes. Même si je suis devenu un adversaire du communisme et de la révolution cubaine, ce n'est sûrement pas un grief à leur faire d'avoir été racistes, pas du tout.

56 Je me suis donc dit qu'il fallait étudier cette affaire, et je me suis mis à lire systématiquement les anthropologues, à étudier les anthropologues américains, Herskovits, Mintz, à lire les ethnologues français, comme Roger Bastide, et sudaméricains, comme Gilberto Freyre au Brésil, ainsi que le Cubain Fernando Ortiz. J'ai tout lu, je me réjouis d'avoir fait ces lectures parce que c'était passionnant. J'ai lu près de deux cents livres à l'époque, dont naturellement ceux de Leiris, concernant l'histoire raciale et les différentes thèses en présence. J'ai étudié en même temps l'histoire de l'Amérique, j'ai lu tout ce qui a été écrit sur la conquête espagnole. J'ai suivi à la trace la notion de race, d'où elle venait. J'ai découvert, du point de vue sémantique, d'où venait le mot « race ». C'est à l'origine un terme de vénerie qu'on n'employait pas à propos des hommes: il y a eu une extension, on l'a appliqué aux hommes, à une époque où il y avait une manie de 
classifier, légitime dans les sciences naturelles, avec Linné, Buffon et tous les autres savants du XVIII ${ }^{e}$ siècle. Mais on a voulu aussi classifier les hommes, au point de voir des « Rouges » ici, là des « Noirs », des «Blancs » : tout cela relevait de la pure fantaisie. J'ai fait le rapprochement avec le carnaval. Un beau jour on a décidé que certains étaient des "Indiens », que les autres étaient des «Noirs", que c'était une façon générique de simplifier cavalièrement les choses. Si on devait tenir compte de chaque ethnie africaine et des religions, on n'en sortait pas. Donc, on avait en face de soi le « Noir » : c'était aussi une façon de discréditer, de diminuer l'être qu'on opprimait. C'est une tentation diabolique qui est venue à l'esprit des colonisateurs d'ajouter ce malheur à tous les autres malheurs de la colonisation et de l'esclavage.

Peu à peu, je suis arrivé à comprendre qu'il y avait dans le mot - c'est pour cela que je dis sémantique et sémiotique - dans cette désignation, ce déguisement ontologique des êtres humains, qui fait peut-être partie de l'histoire générale des signes. Je n'avais pas la formation suffisante pour ébaucher une théorie de la perception. Jusqu'à ce jour, je n'ai pas ébauché une théorie, j'ai émis quelques intuitions que j'ai senties. Je me réjouis de voir un homme comme vous, avec votre savoir, retenir cela, que cela fait partie d'une sémiotique générale. Dans l'ordre de l'histoire des signes, il faut retenir cette tendance qui a conduit à faire de ce qui n'a aucune signification esthétique et morale une valeur, alors que la couleur de la peau peut être considérée comme un avatar de l'acclimatation, de l'adaptation des hommes à différents climats, à différentes régions: une forme d'adaptation à la nature, cela s'arrête là. Autrement, les hommes sont partout les mêmes. Mais la perversion a commencé le jour où on a donné un sens, donc où on a constitué en valeur la couleur elle-même, témoin de la diversité des formes physiques historiques de la condition humaine.

[...]

Je construis la vie comme une combustion. Je n'ai pas une vision manichéenne du monde, mais je pense qu'on doit s'efforcer d'être des hommes et des femmes de bonne combustion, c'est-à-dire fonctionner pour la beauté, pour la bonté, pour la solidarité, pour la fraternité, toutes choses qui sont encore restées loin des côtes d'Haïti, mais dont j'espère qu'elles seront un jour acclimatées dans une démocratie haïtienne.

\section{AUTEUR}

JEAN-LUC BONNIOL

Université Aix-en -Provence, jean-luc.bonniol@wanadoo.fr 